\title{
Estudio experimental y numérico del desplazamiento y rotación de suelo reforzado con geomallas bajo empuje activo
}

\section{Experimental and numerical study of the displacement and rotation of geogrid reinforced soil under active lateral earth pressure}

\section{Lissette Ruiz-Tagle ${ }^{1}$ y Felipe Villalobos ${ }^{2}$}

${ }^{1}$ Constructora Lancuyen Ltda., Barros Arana 492, Of. 63, Concepción, Chile, lruiz-tagle@lancuyen.cl (anteriormente en el Instituto de Geotecnia de la Universidad RWTH-Aachen, Alemania)

${ }^{2}$ Departamento de Ingeniería Civil, Universidad Católica de la Santísima Concepción, Alonso de Ribera 2850, Concepción, Chile, avillalobos@ucsc.cl

Sepresentaunestudio experimentalynumérico de desplazamiento y rotación de suelo sin y con refuerzo de geomallas bajo la acción del empuje activo. Para ello se ha usado la técnica de medición PIV, con la cual se procesan imágenes digitales para la obtención de campos de desplazamiento y rotación. El equipo de ensayo de empuje posee una ventana transparente que permite registrar el movimiento del suelo. Los resultados medidos de movimiento del suelo permiten determinar áreas de suelo que fluyen y no fuyen debido a la acción del empuje activo. De esta manera se puede determinar la posición y geometría de la superficie de falla, la cual se puede comparar con la definida por la teoría de la plasticidad de empuje activo de Rankine. Esta comparación resulta solo posible para el caso sin geomallas, dado que con geomallas la zona de suelo en fluencia cambia significativamente. La presencia de geomallas puede reducir hasta dos veces el volumen de suelo que entra en fluencia debido al empuje activo. Esta conclusión relacionada con la deformación del suelo está en directa relación con la disminución de la tensión de empuje activo al utilizar geomallas como elementos de refuerzo. También se presenta un análisis numérico usando el programa computacional Plaxis para estimar los desplazamientos horizontales. Los resultados numéricos entregan estimaciones que se aproximan a los resultados medidos de desplazamientos horizontales en términos de la forma de la zona de suelo en fluencia. Sin embargo, no son capaces de reproducir en detalle la geometría de la superficie de falla.

Palabras clave: empuje activo, deformación plana, geomalla, desplazamiento horizontal, rotación, imágenes digitales PIV
An experimental and numerical study is presented related to displacement and rotation of soil with and without geogrid reinforcement under the application of active lateral earth pressure. To this end, the measurement technique PIV has been used, with which digital images are processed to obtain displacement and rotation fields. The experimental earth pressure equipment has a transparent window which allows the recording of soil movements. Through the process and analysis of the measured soil movement, soil areas which yielded and did not yield due to the active earth pressure, were defined. In this form, the position and geometry of the failure surface can be determined and compared with the defined by the plasticity theory of active Rankine states. This comparison is only possible for the case without geogrids since the presence of geogrids change significantly the zone of soil yielding. Geogrids can reduce up to twice the volume of soil yielding due to the active lateral earth pressure. This conclusion related with the soil deformation is in direct relation with the active lateral earth pressure reduction when geogrids are used as reinforced elements. In addition, a numerical analysis using the computational program Plaxis is presented to estimate horizontal displacements. The numerical results estimate reasonably well the horizontal displacements measured in terms of the shape of the soil yielding area. However, they do not reproduce in detail the geometry of the failure surface.

Keywords: active earth pressure, plane strain, geogrid, horizontal displacement, rotation, digital images PIV

(Giroud 1986; Koerner 1986; Müller-Rochholz 2008). Se suma a ello que en comparación a estructuras de contención tradicionales como muros gravitacionales, L o $\mathrm{T}$, los métodos constructivos son económicamente más atractivos debido a su flexibilidad y versatilidad en su
Dentro de la Ingeniería Geotécnica ya es reconocida la significativa contribución de las geomallas en el mejoramiento de la capacidad resistente y disminución de la deformación de suelos de fundación y rellenos artificiales 
instalación, además se les atribuye una menor huella de carbono (O'Riordan et al. 2011).

El estudio del empuje activo de rellenos compactados y reforzados sobre muros de contención se ha basado principalmente en la teoría de la plasticidad, aplicando los estados de Rankine de empuje (Jones 1984, 1996). Sin embargo, mediciones en muros de contención de $4.65 \mathrm{~m}$ y $6 \mathrm{~m}$ de altura reforzados con geomallas realizadas por Berg et al. (1986) indicaron que las tensiones horizontales de empuje resultaron menores a

las determinadas mediante la teoría de Rankine. La incorporación de geomallas cambia significativamente la distribución lineal del empuje activo con la profundidad. Más recientemente, resultados presentados por Pachomow et al. (2007) de ensayos de empuje en un muro de $3.5 \mathrm{~m}$ reforzado con geomallas, incluyendo resultados recolectados de una amplia base de datos, hace posible notar diferencias importantes con la distribución teórica lineal de empujes. Estas diferencias se han visto reflejadas en parte en las recientes actualizaciones de las recomendaciones de EBGEO (2009), donde se asume que el empuje que actúa sobre una pared reforzada con geomallas es menor que lo que se planteaba en la versión anterior de 1997.

En la literatura técnica es difícil encontrar resultados experimentales de desplazamiento de suelo reforzado con geomallas y con sobrecarga $\sigma$. La poca información existente ha sido obtenida numéricamente mediante el método de elementos finitos (Pachomow et al. 2007). Es por ello que esta investigación se focalizó principalmente en el estudio de la variación del desplazamiento del suelo retenido detrás de una estructura de contención. Para ello se ha utilizado una técnica de tratamiento de imágenes digitales llamada PIV, además de simulaciones usando el programa computacional Plaxis. Este trabajo se encuentra enmarcado dentro del proyecto de investigación sobre respuesta tensodeformacional de suelos reforzados con geomallas desarrollado en la Universidad de Aachen.

\section{Equipamiento experimental}

El equipo de ensayo utilizado y mostrado en la Figura 1, ha sido diseñado y construido por Ruiken et al. (2010a, 2010b y 2011). En este artículo se analiza desde el punto de vista de los desplazamientos y rotaciones, el trabajo experimental presentado por RuizTagle y Villalobos (2011). En ese trabajo ellos analizan los resultados experimentales basándose en la variación de las tensiones horizontales de empuje de suelo reforzado con geomallas, no en los desplazamientos ni rotaciones. Se recomienda revisar esa publicación para obtener mayor información sobre el equipo de ensayo, preparación de las muestras y características principales de la arena de Marienberg y de la geomalla usada.

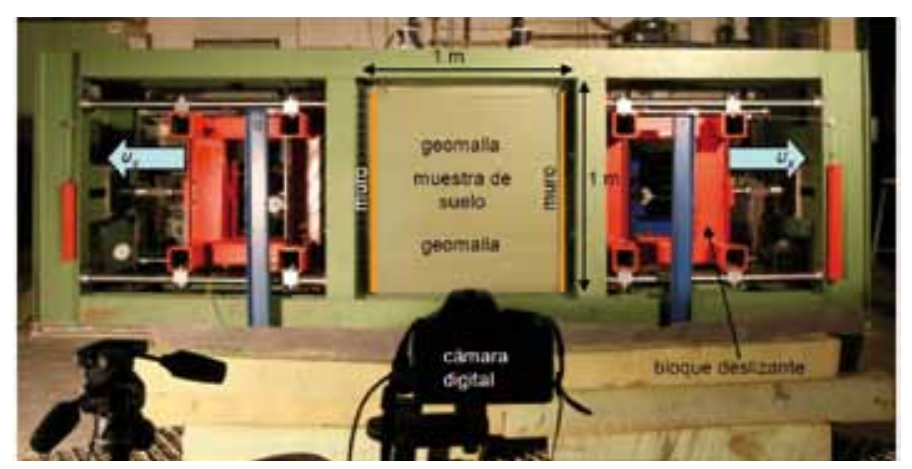

Figura 1: Equipo de ensayo de empuje sobre una muestra con dos geomallas demarcadas con arena negra junto al vidrio y emplazamiento de las cámaras digitales para análisis de desplazamientos PIV

Las dimensiones de la muestra de suelo son de $1 \mathrm{~m}$ de alto, $1 \mathrm{~m}$ de largo y $0.45 \mathrm{~m}$ de ancho (Figura 1). Existen dos muros independientes a cada extremo de la muestra de $1 \mathrm{~m}$ de alto y $0.45 \mathrm{~m}$ de ancho. Cada muro puede ser desplazado por un bloque que desliza sobre barras horizontales. La aplicación de empuje activo se obtiene a partir de un desplazamiento $u_{x}$ del bloque, tal como se indica en la Figura 1. Si bien es cierto se puede considerar como un modelo de muro a escala, también puede ser considerado como una parte de un muro como se muestra en la Figura 2.

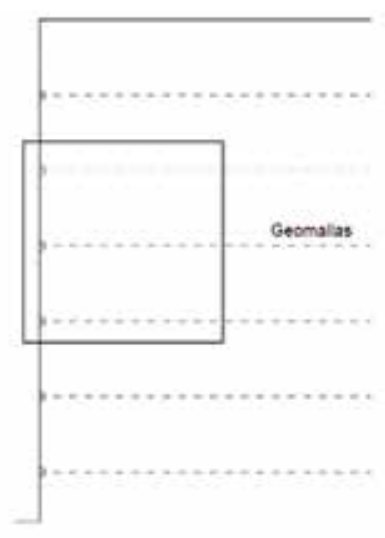

Figura 2: Prototipo de estructura de contención de suelo reforzado con geomallas, mostrando una parte de suelo a estudiar en el laboratorio 


\section{Determinación de la deformación del suelo}

Para estudiar la deformación del suelo reforzado con geomallas se utilizó el método óptico conocido como PIV por su sigla en inglés (Particle Image Velocimetry). Este método permite determinar los movimientos y las rotaciones de las partículas de suelo en un plano visible, generalmente detrás de un vidrio o material transparente. Para ello se requiere el uso de una cámara digital que registre cada cierto intervalo de tiempo un marco fijo de enfoque para así detectar el movimiento de los granos. En el presente estudio los granos de arena se mueven producto de la acción del empuje activo provocado por el desplazamiento de la pared, por ejemplo, en secuencias de $0,0.1,0.2,0.5,1,5,10 \mathrm{~mm}$ ( $0 \mathrm{~mm}$ es el caso en reposo). Ruiz-Tagle y Villalobos (2011) encontraron que el empuje activo se desarrolla plenamente para un valor de $0.2 \mathrm{~mm}$, interpretado cuando las tensiones horizontales medidas igualan a las calculadas con la teoría de Rankine para un suelo sin reforzar. La visualización de la geomalla es lograda con la ayuda de una delgada línea de arena oscura, la cual se esparció a lo largo de la geomalla junto al cristal (ver Figura 1 y 3 ). La Figura 3 muestra un detalle del suelo deformado en la parte superior, se observa un asentamiento y una dislocación del suelo sobre la geomalla.

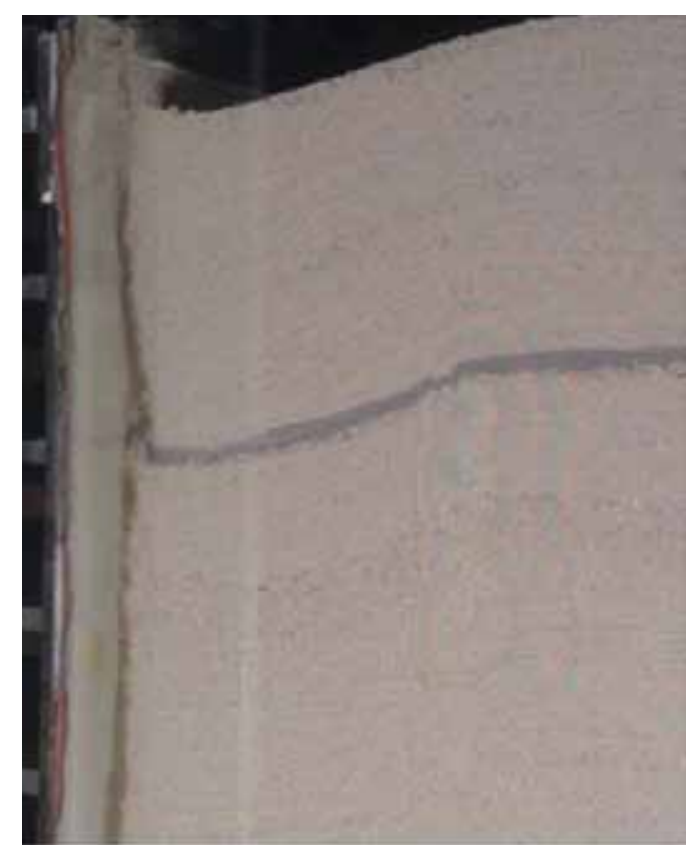

Figura 3: Detalle superior de deformación

Durante los ensayos de empuje se tomaron fotos a toda la muestra y en algunos casos se utilizó otra cámara para determinar con más detalle un área en particular al mismo tiempo. El posicionamiento de la cámara fue en cada ensayo el mismo, con una altura desde el suelo al centro del objetivo de $0.7 \mathrm{~m}$ con el fin de fotografiar la totalidad del área frontal de la muestra de suelo. La segunda cámara se emplazó de acuerdo al área de interés. Las fotos fueron tomadas en intervalos de desplazamiento horizontal de la pared $u_{x}=0.1 \mathrm{~mm}$. Para obtener información sobre el método PIV, se recomienda revisar White et al. (2003) y White y Bolton (2004).

\section{Resultados en suelo sin refuerzo}

Como una primera etapa de estudio del empuje activo se realizaron mediciones con suelo sin geomallas de refuerzo. La Figura 4 muestra la variación del campo de desplazamientos y rotaciones de partículas de suelo obtenido de las mediciones usando el método PIV para cuatro niveles de desplazamiento horizontal $u_{x}$ de la pared; 0, 0.6, 3 y $20 \mathrm{~mm}$ respectivamente. El campo de desplazamientos y rotaciones se interpreta por medio de la dirección y magnitud relativa de vectores de desplazamiento y como rotación en el sentido horario o antihorario y con una magnitud relativa cualitativa mostrada con colores. Para un desplazamiento de la pared $u_{x}$ de $0.6 \mathrm{~mm}$ se visualiza levemente una demarcación de una zona de suelo en movimiento, a diferencia de para $u_{x}=$ $0.2 \mathrm{~mm}$, valor para el cual Ruiz-Tagle y Villalobos (2011) determinan la movilización del empuje activo a partir de las mediciones de la variación de tensiones. Esta diferencia se puede deber al efecto del empuje en reposo, el cual aplica empujes iniciales mayores al activo, pero desplazamientos nulos, o sea no afecta en la medición de desplazamientos.

Los resultados del método PIV permiten observar claramente el desarrollo de una zona de mayor rotación para $u_{x} \geq 3 \mathrm{~mm}$, la cual delimita una superficie de falla. De acuerdo a la teoría de la plasticidad la superficie de falla es definida por una recta que forma con la horizontal un ángulo $\theta=45^{\circ}+\varphi / 2$. Dado que la arena fue ensayada con una densidad relativa de $93 \%$ y un $\varphi_{\mathrm{tx}}=40^{\circ}$, resulta $\theta=65^{\circ}$. Sin embargo, el ángulo obtenido del ensayo es de aproximadamente $75^{\circ}$, lo cual indica que el estado de equilibrio plástico o estado activo de Rankine es una solución que involucra mayor área de suelo plastificado. Además se observa que la superficie de falla tiende a ser vertical en la parte superior. Esto tal vez sea debido al mayor desplazamiento horizontal y vertical (asentamiento) en la parte superior en comparación a la parte inferior del muro. 


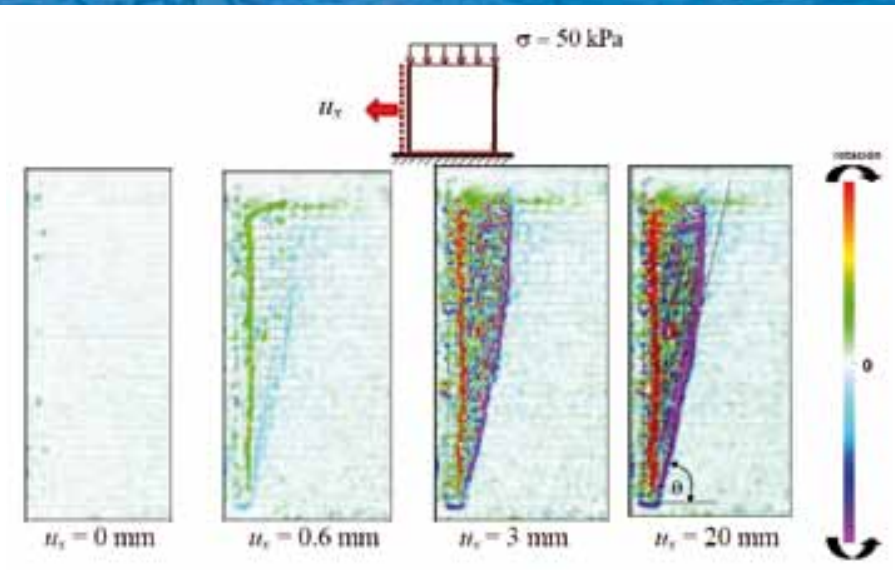

Figura 4: Rotación de partículas para distintos desplazamientos de la pared para una sobrecarga de $50 \mathrm{kPa}$

\section{Resultados en suelo reforzado}

A continuación se presentan resultados de los campos de desplazamientos y rotaciones de partículas de suelo reforzado detrás de una pared en movimiento para generar empuje activo. La muestra de suelo ha sido reforzada con dos, tres y cuatro geomallas respectivamente. Dentro de la muestra de suelo de $1 \mathrm{~m}$ de altura se colocaron dos geomallas a $0.3 \mathrm{~m}$ y $0.7 \mathrm{~m}$ desde el nivel superior; a $0.2 \mathrm{~m}, 0.5 \mathrm{~m}$ y $0.8 \mathrm{~m}$ para tres geomallas y a $0.1 \mathrm{~m}, 0.35 \mathrm{~m}, 0.65 \mathrm{~m}$ y 0.9 $\mathrm{m}$ para cuatro geomallas. La Figura 5 muestra el campo de desplazamientos representado por vectores y la rotación de partículas representada por colores, después que la pared se ha desplazado $10 \mathrm{~mm}$. Se puede observar que las geomallas contienen o evitan que la superficie de falla se extienda o involucre más suelo (ver flecha superior para comparar). De esta manera las dos geomallas reducen el área o volumen de suelo desplazado detrás de la pared. La superficie de falla adopta una forma curva entre las geomallas. Esto es similar a la distribución curva de tensiones horizontales de empuje entre dos geomallas presentado por Ruiz-Tagle y Villalobos (2011). Ellos plantean que la disminución del empuje se asocia a la formación de arcos de tensiones entre geomallas, donde la diferencia de empuje es transmitida y tomada por las geomallas.

Los vectores de movimiento indican que los granos de arena ubicados más próximos a la pared, son los que tienen una mayor magnitud relativa de movimiento. Este movimiento relativo de los granos se reduce en la medida que se alejan de la pared y se aproximan a la superficie de falla. La dirección de los vectores indica que existe una importante componente vertical, igual o tal vez mayor que la componente horizontal del movimiento de los granos. El movimiento vertical de los granos tiende a ser mayor en la parte superior de la muestra. Y la componente horizontal del movimiento relativa a la vertical aumenta en la medida que los granos se encuentran más cerca de la base del muro.

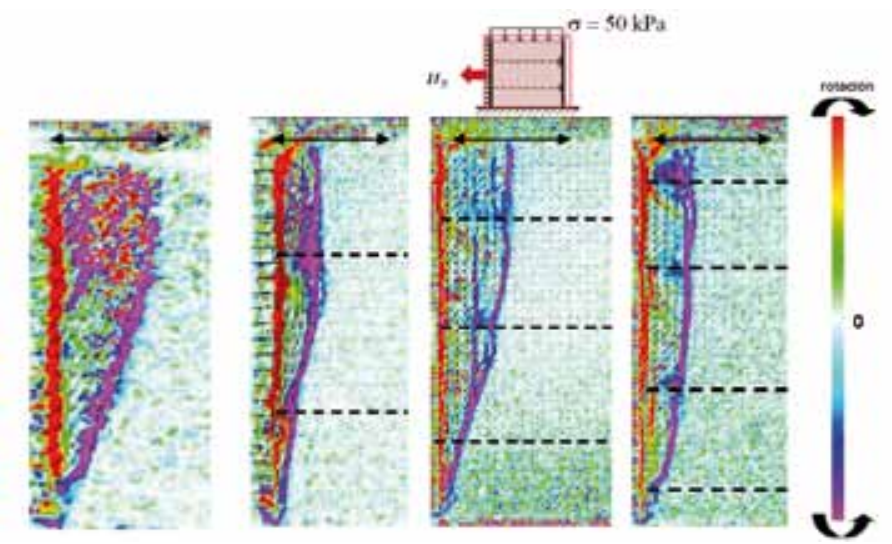

Figura 5: Rotación de partículas sin geomalla y con dos, tres y cuatro geomallas, sobrecarga de $50 \mathrm{kPa}$ y $u_{x}=10 \mathrm{~mm}$

A continuación se compara la deformación del suelo junto a la pared para el caso con y sin geomalla. En la Figura 6a se muestran detalles de $0.3 \mathrm{~m}$ x $0.2 \mathrm{~m}$ ubicados en la geomalla superior para $u_{x}=10 \mathrm{~mm}$ y una sobrecarga $\sigma$ $=50 \mathrm{kPa}$. En la Figura $6 \mathrm{~b}$ se muestran resultados de desplazamiento horizontal obtenidos con el método PIV usando fotos de alta resolución (15 megapixels), para el caso con y sin geomalla. El color azul indica la ausencia de desplazamiento horizontal y el color rojo indica la existencia de valores máximos de desplazamiento horizontal. Con estos resultados es posible establecer que la geomalla ha disminuido prácticamente a la mitad el ancho de la zona sometida a deformaciones de fluencia (zona no azul). También es posible distinguir que la geomalla cambia la variación uniforme de los desplazamientos horizontales observable en el caso sin geomalla (cambio gradual de colores de izquierda a derecha).

La Figura 6c muestra nuevamente este mismo detalle, pero en término de vectores de desplazamiento (para el caso sin geomalla) y en término de rotación de partículas. En el caso sin geomalla se desarrolla una única superficie de falla. Sin embargo, en el caso con geomalla, además de la superficie de falla principal vertical, se observa el desarrollo de otras dos líneas de falla casi paralelas. También es posible observar el desarrollo de una línea de falla entre la pared y la geomalla y que baja en $45^{\circ}$ hasta tocar la superficie de falla vertical. A pesar que la geomalla no está fija a la pared se transmiten tensiones de corte entre la geomalla y el suelo, lo cual forma un triángulo de suelo sobre y bajo esta superficie de falla 
en $45^{\circ}$. Notar que el color lila indica rotación horaria y el color rojo antihoraria. Por lo tanto el triángulo superior de suelo actúa como una cuña activa desplazándose hacia abajo y el triángulo inferior es desplazado por esta cuña horizontalmente hacia la izquierda. Esto complementa las observaciones realizadas por Ruiken et al. (2010b).

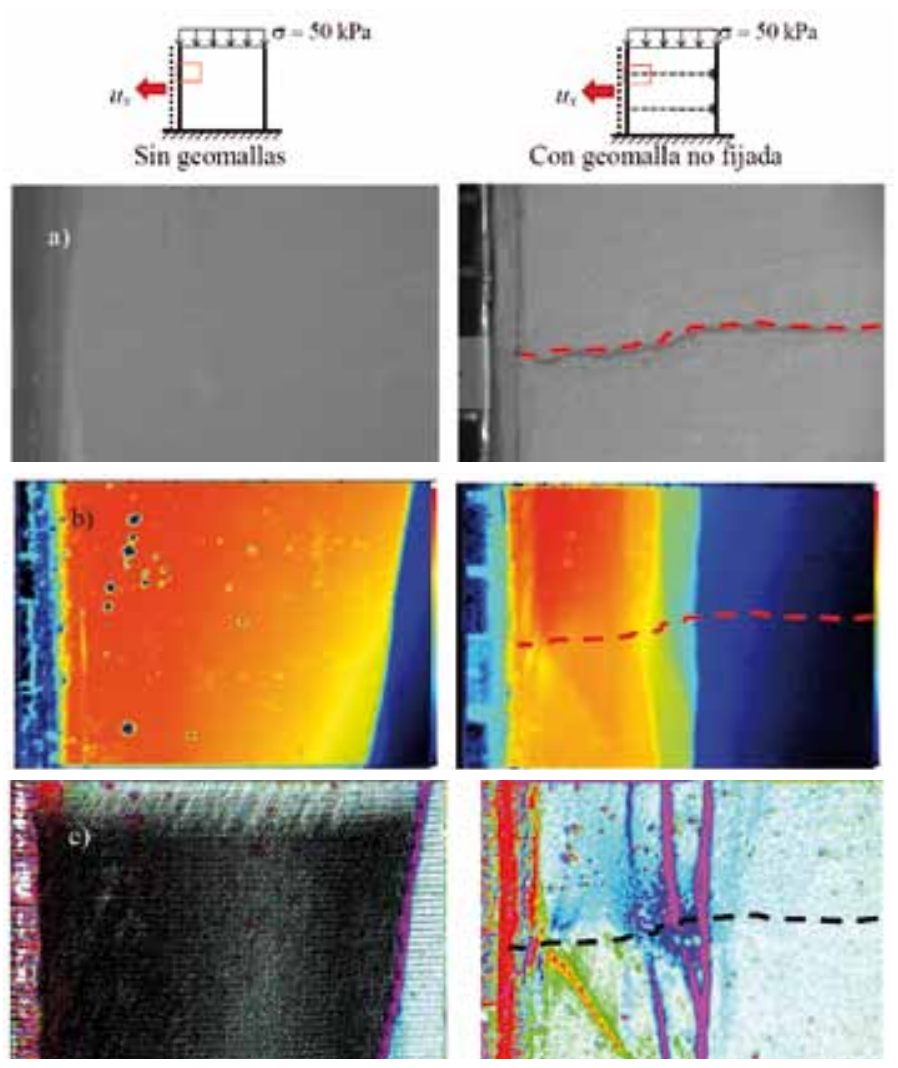

Figura 6: Detalle del suelo junto a la pared para $u_{r}=10 \mathrm{~mm}$ y sobrecarga $\sigma=50 \mathrm{kPa}$, a) suelo deformado, b) desplazamiento horizontal obtenido con PIV y c) vectores de desplazamiento y rotación de partículas

\section{Análisis numérico}

Se utilizó el programa computacional Plaxis de elementos finitos bidimensionales para estudiar la deformación del suelo. La modelación se realiza para una condición de deformación plana con 15 nodos por elementos. Se consideró una densidad de malla media dado que se logra una exactitud de $98 \%$, la cual es suficiente para obtener buenas aproximaciones en los cálculos. Mallas finas a muy finas requieren mayor consumo de recursos y tiempo computacional para alcanzar un incremento menor en exactitud.

Se estudiaron las condiciones de borde con el fin de emular los ensayos de laboratorio. En primer lugar se tuvo en cuenta el efecto de interacción entre suelo y estructura, dado que se pueden generar movimientos relativos en las superficies de contacto. Sin embargo, en un análisis de elementos finitos que involucre suelo, el uso de elementos continuos con compatibilidad de desplazamientos podría no permitir estos movimientos relativos. Por lo tanto al usar el método de elementos finitos se debe implementar la compatibilidad nodal, la cual obligue a un movimiento en conjunto de los elementos adyacentes. Para incorporar la compatibilidad nodal, Plaxis posee la opción interface para elementos usados en la modelación de superficies de contacto entre suelo y estructura. Así se posibilita modificar el comportamiento constitutivo de las superficies de contacto (fricción entre distintos materiales), y además se permiten los movimientos relativos (deslizamientos y separación).

La Figura 7 muestra el resultado de modelar sin interface las paredes. Notar que a pesar del empuje activo aplicado, el suelo no se asienta donde tiene contacto con la pared, contrariamente a lo observado en los ensayos (ver Figura 3). Esto es debido a las condiciones de borde impuestas en la modelación, producto de la fricción entre el suelo y la pared, la cual no permite que el suelo se deslice o despegue de la pared.
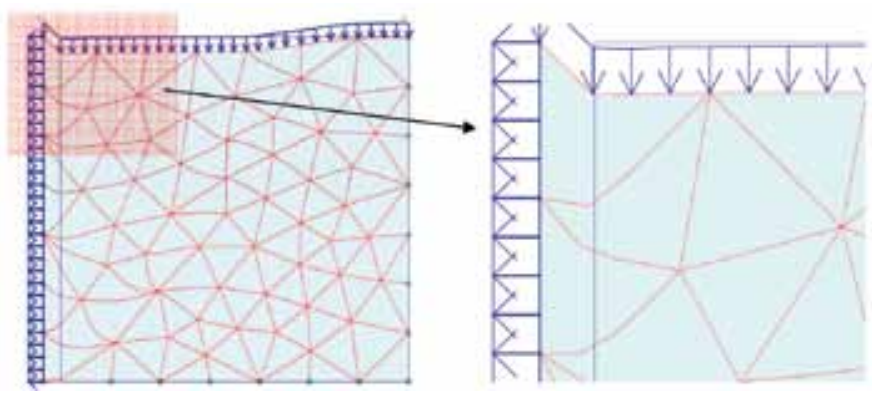

Figura 7: Malla deformada con suelo sin geomalla y sin interface

Cuando se utiliza un valor de interface $\mathrm{R}_{\text {inter }}=1$, el efecto de adherencia entre el suelo y la pared desaparece. Sin embargo, aparece un vacío entre la pared y el suelo como se observa en la Figura 8. En vista de esto se consideró en las simulaciones computacionales un valor $\mathrm{R}_{\text {inter }}=0.3$, el cual representa de mejor manera el ángulo interfaz de fricción de contacto entre el suelo y la pared de $2.6^{\circ}$. El asentamiento también varía con el valor de $\mathrm{R}_{\text {inter }}$ pero se considera que con $\mathrm{R}_{\text {inter }}=0.3$ se obtienen asentamientos similares a los obtenidos experimentalmente. Valores menores a 0.3 no son adecuados porque el suelo comienza a entrar o sobrepasar la pared, lo cual no es real representando incompatibilidad de deformaciones. 


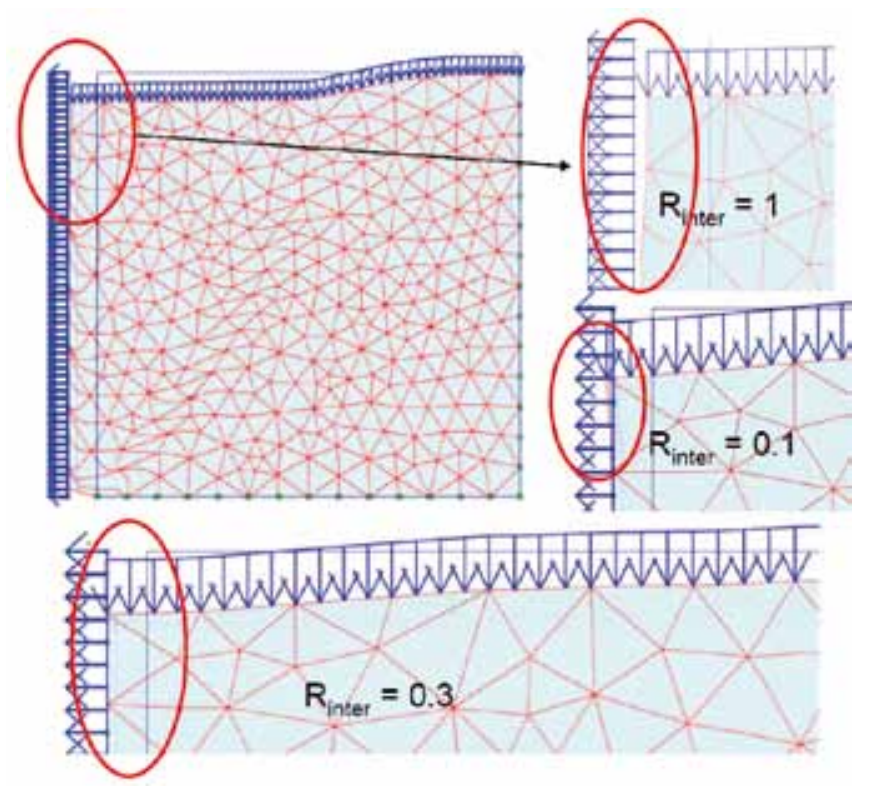

Figura 8: Malla deformada para suelo no reforzado mostrando efecto de borde en contacto muro y suelo próximo a la superficie para interface $\mathrm{R}_{\text {inter }}=1,0.3$ y 0.1

Los valores de los parámetros geotécnicos del suelo determinados en el laboratorio son un peso unitario seco $\gamma_{\mathrm{d}}=17.3 \mathrm{kN} / \mathrm{m}^{3}$ y un ángulo de fricción interna $\varphi_{\mathrm{tx}}=$ $40^{\circ}$. Se asumió un módulo de elasticidad $\mathrm{E}=10^{5} \mathrm{kPa}$ y un coeficiente de Poisson de 0.25. Para la simulación se modificó el ángulo de fricción de $40^{\circ}$ obtenido en ensayos triaxiales por un valor igual a $46^{\circ}$. Esto con el objeto de reproducir condiciones de deformación plana de acuerdo a lo sugerido por Lee (1970). Debido a esto se modificó levemente el módulo de elasticidad a $\mathrm{E}=1.04 \cdot 10^{5} \mathrm{kPa}$ y se incorporó una pequeña cohesión de $0.2 \mathrm{kPa}$.

El valor de la rigidez de la geomalla usada en la modelación con Plaxis fue EA $=2100 \mathrm{kN} / \mathrm{m}$. La Figura 9a muestra resultados de desplazamiento horizontal para el caso sin geomallas y la Figura $9 \mathrm{~b}$ para el caso con dos geomallas. Se puede observar que estos resultados numéricos reproducen el efecto de reducción de área de la zona de suelo en fluencia debido al empuje activo. Además se forman los arcos de desplazamiento junto a la pared. Al comparar la Figura 9 con las Figuras 5 y 6 se nota que la simulación con Plaxis no es capaz de reproducir la pendiente casi vertical de la superficie de falla en el caso sin y con geomalla. El análisis numérico es una idealización dado que asume el suelo como un continuo, cuando el suelo es en verdad un material granular. Mayores estudios son necesarios para modelar numéricamente los desplazamientos y rotaciones del suelo producto del empuje activo.
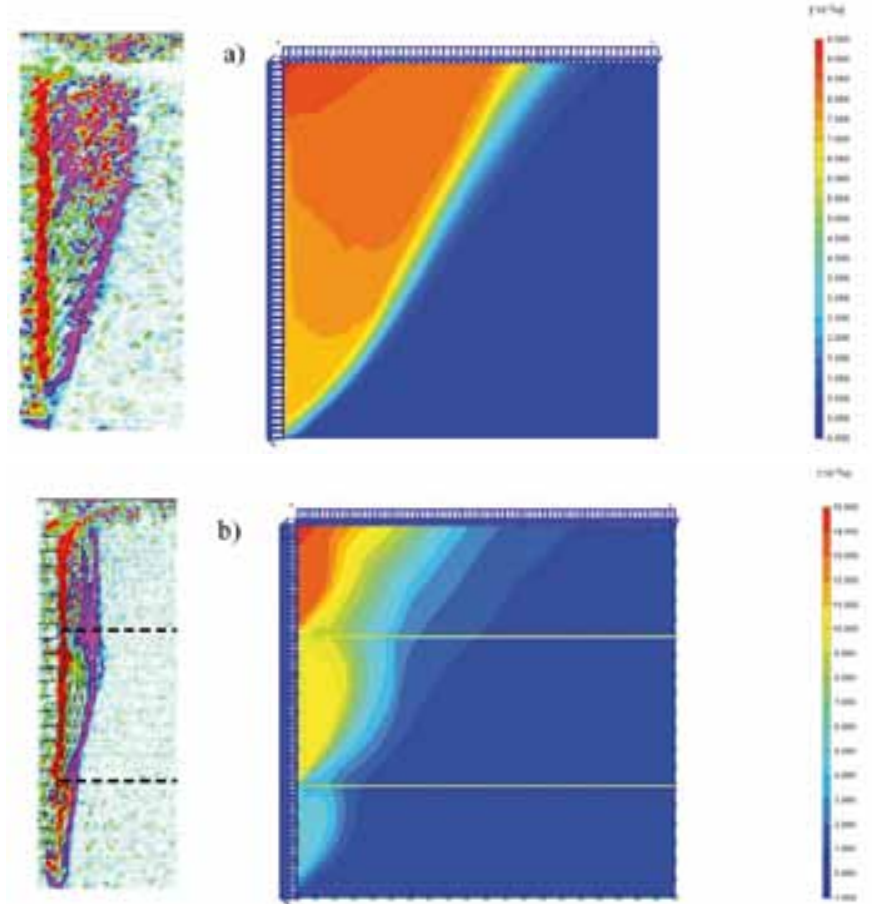

Figura 9: Desplazamiento horizontal del suelo para $\sigma=50 \mathrm{kPa}$ y $u_{x}=10 \mathrm{~mm}$, a) sin geomalla y b) con dos geomallas (se muestran también los resultados de la Figuras 5 y 6 por comparación)

\section{Conclusiones}

Se han presentado resultados de desplazamiento del suelo detrás de una pared de contención, la cual es sometida a empuje activo. Esto ha sido posible mediante el uso de la técnica PIV, la cual permite generar campos de desplazamientos y rotaciones de partículas en movimiento. El análisis del campo de desplazamientos relativos y rotaciones relativas se realizó de manera cualitativa por medio de vectores y colores respectivamente, es decir, no se obtuvieron magnitudes absolutas en unidades ingenieriles.

Los resultados más destacados son la reducción del área de suelo en fluencia cuando se ocupan geomallas, reducción que puede llegar a ser hasta la mitad si se compara con el caso sin geomallas. Esto explica la reducción de las tensiones horizontales de empuje medidas con el mismo equipo de ensayo y reportadas por Ruiken et al. (2011) y Ruiz-Tagle y Villalobos (2011). Esta reducción de tensiones horizontales de empuje se debe a que la diferencia es tomada por las geomallas. Así se induce menos empuje sobre el muro dado que el volumen de suelo en fluencia es menor.

Se ha presentado un análisis numérico usando el programa 
computacional Plaxis. Los resultados iniciales presentan una concordancia general con los resultados medidos en cuanto a los desplazamientos horizontales sin y con geomallas. Sin embargo, existen diferencias respecto a la forma de la zona de fluencia, por ejemplo en la pendiente de la superficie de falla. Por lo tanto se requieren mayores estudios para mejorar los resultados numéricos.

\section{Agradecimientos}

Este trabajo fue posible gracias a la beca otorgada por el DAAD y la Universidad Católica de la Santísima Concepción a la primera autora. La primera autora agradece especialmente al Dipl.-Ing. Axel Ruiken por su apoyo y supervisión y también al personal del laboratorio de Geotecnia de la Universidad RWTH-Aachen.

\section{Referencias}

Berg,R.R.,Bonaparte, R.,Anderson, R.P. andChouery, V.E.(1986). Design, construction and performance of two geogrid reinforced soil retaining walls. Proceedings of the Third International Conference on Geotextiles, Vienna Austria, Vol. 2, 401-406

EBGEO (2009). Empfehlungen für den Entwurf und die Berechnung von Erdkörpern mit Bewehrungen aus Geokunststoffen. Deutsche Gesellschaft für Geotechnik

Giroud, J.-P. (1986). From geotextiles to geosynthetics: a revolution in Geotechnical Engineering. Proceedings of the Third International Conference on Geotextiles, Vienna Austria, Vol. 1, 1-18

Jones, C.J.F.P. (1996). Earth reinforcement and soil structures. Thomas Telford

Jones, C.J.F.P. (1984). Design and construction methods. Symposium on Polymer Grid Reinforcement in Civil Engineering. ICE London

Koerner, R.M. (1986). Designing with Geosynthetics. PrenticeHall
Lee, K. L. (1970). Comparison of plane strain and triaxial test on sand. Journal of the Soil Mechanics and Foundation Division ASCE 96, No. SM3, 901-923

Müller-Rochholz, J. (2008). Geokunststoffe im Erd- und Verkehrswegebau. Werner Verlag

O’Riordan, N., Nicholson, D., Hughes, L. and Phear, A. (2011). Examining the carbon footprint and reducing the environmental impact of slope engineering options. Ground Engineering 44, No 2, 28-30

Pachomow, D., Vollmert, L. und Herold, A. (2007). Der Ansatz des horizontalen Erddrucks auf die Front von KBEKronstruktionen. J. Geotechnik Sonderheft, 129-136

Ruiken, A., Ziegler, M., Vollmert, L. and Höhny, S. (2011). Investigation of the compound behavior of geogrid reinforced soil. Submitted to the Fifteenth European Conference on Soil Mechanics and Geotechnical Engineering, Athens

Ruiken, A., Ziegler, M., Vollmert, L. and Duzic, I. (2010a). Recent findings about the confining effect of geogrids from large scale laboratory testing. $9^{\text {th }}$ International Conference on Geosynthetics, Guarujá, Brazil

Ruiken, A., Ziegler, M., Ehrenberg, H. and Höhny, S. (2010b). Determination of the soil confining effect of geogrids. XIV ${ }^{\text {th }}$ Danube-European Conference on Geotechnical Engineering, Bratislava, Slowak Republic

Ruiz-Tagle, L. and Villalobos, F.A. (2011). Experimental study of the lateral earth pressure on retaining structures in soils reinforced with geogrids. Submitted to Revista Ingeniería de Construcción

White, D. J., Take, W.A. and Bolton, M.D. (2003). Soil deformation measurement using particle image velocimetry (PIV) and photogrammetry. Géotechnique 53, No. 7, 619-631

White, D. J. and Bolton, M.D. (2004). Displacement and strain paths during plane-strain model pile installation in sand. Géotechnique 54, No. 6, 375-397 


\section{Requerimientos de publicación}

1. Los autores deben indicar la categoría de clasificación del artículo, la cual puede ser: artículo técnico (investigación), nota técnica (artículo corto), informe técnico (de diseños, construcciones, monitoreos, etc.), discusión o comentario (de material publicado previamente). Los artículos enviados deberán ser originales y no haber sido publicados antes ni estar en proceso de revisión por otra revista. Ellos pueden ser escritos en inglés o castellano y deberán ser enviados en versión electrónica en formato word o pdf. Los artículos se envían al Editor Dr. Felipe Villalobos vía correo electrónico a oyp@ucsc.cl (preferido) o en CD vía correo postal a Departamento de Ingeniería Civil, Universidad Católica de la Santísima Concepción, Alonso de Ribera 2850, Casilla 297, Concepción, Chile. Deberá incluirse en el envío la afiliación, dirección postal y electrónica de todos los autores. El Editor podrá requerir revisiones y correcciones antes de tomar una decisión final de aceptación o rechazo del artículo. Una vez que el artículo es recibido conforme a las guías de más abajo, éste será enviado a revisores externos, expertos en los temas específicos que trata el artículo. Los autores tienen la responsabilidad de reconocer y declarar cualquier conflicto de intereses que pudiera sesgar su trabajo, por ejemplo, indicando fuentes de financiamiento y apoyo.

2. El artículo deberá tener una extensión máxima de 8000 palabras, no se exige formato (tipo ni tamaño de letra, espaciamiento, etc.) sólo se pide claridad tanto en lo escrito, o sea, buena redacción con una secuencia lógica de lo que se presenta, como en las figuras, gráficos, tablas y fotos. El formato usado en la revista se encuentra disponible en www.oyp.cl/normas.pdf para quienes quieran seguir el formato.

3. Los artículos deberán tener en su primera página el título con el nombre y apellido del o de los autores, su afiliación, dirección postal y e-mail. El título debe estar en castellano e inglés.

4. El resumen o abstract no debe exceder las 250 palabras y debe apuntar a los aspectos claves del artículo y dar una síntesis de las aplicaciones y conclusiones más importantes que el artículo entrega. El abstract debe ser una traducción correcta del resumen al inglés. Se deben incluir palabras claves tanto en castellano como en inglés.

5. Se usa el sistema Harvard para las referencias. Éstas deben estar completas y correctas. En el texto, las referencias deben citarse como Martínez (2008) o (Goodell y Warren, 2006). Para más de dos autores deben indicarse como (Van Abeelen et al., 2008). Al autor de un mismo año deben diferenciarse usando 2008a, 2008b, etc. El estilo de las referencias debe seguir uno de los esquemas siguientes:

Cepeda, M. (2006). Un nuevo modelo para la estimación del tiempo de espera en paraderos de transporte público. Obras y Proyectos 2 (1)

Wichtmann, T., Niemunis, A., Triantafyllidis, Th. and Poblete, M. (2005). Correlation of cyclic preloading with the liquefaction resistance. Soil Dynamics and Earthquake Engineering 25 (12)

Lunne, T., Robertson, P.K. and Powell, J.J.M. (1997). Cone Penetration Testing in Geotechnical Practice. Spon Press

6. Las tablas y fórmulas (ecuaciones) deberán ser numeradas secuencialmente en el mismo orden en que aparecen en el texto, con números arábigos y haciendo referencia a ellas como: Tabla 1, Tabla 2, Fórmula (1), Fórmula (2)...etc, según corresponda. Éstas deben ser introducidas dentro del texto en el mismo orden en que son referenciadas. En el caso de las tablas, el título debe colocarse en la parte superior y no deben duplicar los resultados presentados en gráficos. El sistema de unidades a emplear será el Sistema Internacional (SI).

7. Las figuras pueden incluir gráficos, esquemas y diagramas. Las figuras deben enviarse en una condición lista para su publicación, con calidad de impresión láser. Si son fotografías, deben ser de alta calidad. Deben numerarse en forma secuencial, en el mismo orden en que son referenciadas en el texto como: Figura 1, Figura 2, Foto 1, Foto 2, etc. y su título deberá colocarse en la parte inferior. Deberán ser incluidas dentro del texto según sean citadas.

8. Las opiniones vertidas en los artículos publicados en Obras y Proyectos son de exclusiva responsabilidad del autor o los autores de los mismos y no reflejan necesariamente los puntos de vista del Departamento de Ingeniería Civil de la Universidad Católica de la Santísima Concepción.

9. El envío de un artículo a la revista Obras y Proyectos implica que representa un trabajo original, no publicado previamente y no considerado para publicación en ninguna otra parte. Los autores aceptan transferir el derecho de autor de sus artículos a la Editorial siempre y cuando el artículo sea aceptado para publicación. El derecho de autor cubre los derechos exclusivos para la reproducción y distribución del artículo, incluyendo re-impresiones, reproducciones fotográficas, microfilms, escaneo de documentos o cualquier reproducción de naturaleza similar así como traducciones. Permisos para usar figuras deben ser obtenidos por el autor en forma previa al envío del artículo. 